\title{
Gating of trigemino-facial reflex from low-threshold trigeminal and extratrigeminal cutaneous fibres in humans
}

\author{
Alessandro Rossi, Chiara Scarpini
}

\begin{abstract}
Changes in the size of the test components (R1 and R2) of the trigemino-facial reflex were studied after electrical subliminal conditioning stimulation were applied to the trigeminal, median and sural nerves. After conditioning activation of the trigeminal nerve (below the reflex threshold), the early $R 1$ reflex component showed phasic facilitation, peaking at about $50 \mathrm{~ms}$ of interstimulus delay, followed by a long-lasting inhibition recovering at $300-400 \mathrm{~ms}$. The same conditioning stimulation resulted in a monotonic inhibition of the late $R 2$, starting at $15-20 \mathrm{~ms}$, with a maximum at $100-150 \mathrm{~ms}$ and lasting $300-400 \mathrm{~ms}$. Intensity threshold for both the $R 1$ and $R 2$ changes ranged from 0.90 to 0.95 times the perception threshold. A similar longlasting inhibition of the $R 2$ reflex response was also seen after conditioning stimulation applied to low-threshold cutaneous afferents of the median and sural nerves. The minimum effective conditioning-test interval was 25-30 ms and 40-45 ms respectively and lasted $600-700 \mathrm{~ms}$. By contrast the early $\mathbf{R} 1$ reflex response exhibited a slight longlasting facilitation with a time course similar to that of the $R 2$ inhibition. The threshold intensity to obtain facilitation of the $R 1$ and inhibition of the $R 2$ test responses after conditioning volley in the median and sural nerves was similar and ranged from 0.9 to 1.2 times the perception threshold. These results demonstrate that low-threshold cutaneous afferents from trigeminal and limb nerves exert powerful control on trigeminal reflex pathways, probably via a common neural substrate. There is evidence that, in addition to any post-synaptic mechanism which might be operating, presynaptic control is a primary factor contributing to these changes.
\end{abstract}

$(\Im$ Neurol Neurosurg Psychiatry 1992;55:774-780)

Laboratory of Neurophysiology, Institute of Neurological Sciences, University of Siena, Italy

A Rossi

C Scarpini

Correspondence to:

Dr Rossi, Istituto di Scienze Neurologiche, Universita' di

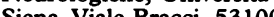
Siena, Italy.

Received 30 August 1991 and in revised form 2 December 1991.

Accepted 15 January 1992

The trigemino-facial reflex has been largely used as a test reflex for studying the organisation and integrity of brainstem reflex circuits. The reflex response of theorbicularis oculi muscles to unilateral electrical stimulation of the first trigeminal (TR) branch shows two main components: an early and brief component (Rl) and a later prolonged component (R2). ${ }^{1-3}$ Under appropriate experimental conditions a third reflex component (R3) can also be constantly obtained. ${ }^{4}$ Many physiological studies suggest that the short and long latency components arise from different but parallel neural pathways. ${ }^{5-12}$

Since the original description by Kimura, ${ }^{13}$ the paired shock technique has been largely employed to study the recovery cycle of the TR reflex components, both in physiological and pathological conditions. ${ }^{14-22}$ In all these studies conditioning stimuli were above the threshold for reflex responses. This caused difficulties in interpretation of the results: a) at conditioning-test intervals shorter than $80-100 \mathrm{~ms}$ the reflex response evoked by the conditioning stimulus overlaps the test reflex, obscuring the effect of the conditioning volley on the latter; b) when the motor neurons are fired in the conditioning reflex response, a long lasting depression of their excitability, due to postspike after-hyperpolarisation, can be expected; c) in addition to any direct effect produced by the conditioning stimulus, there may also be those due to afferent reactivation during muscular contraction; d) finally, using high intensity conditioning stimuli, no conclusion can be reached on the type of afferent fibres responsible for the effects observed on test responses.

In this study, graded subliminal conditioning stimuli were applied to TR afferents and the respective effects on the early $R \mathbf{l}$ and late $\mathrm{R} 2$ components of the trigemino-facial reflex were evaluated. The same TR reflex components were also studied after conditioning activation of low threshold cutaneous afferents arising from median ( $\mathrm{MN}$ ) and sural (SR) nerves.

\section{Methods}

The experiments were performed on 5 adult volunteers (30-37 years), all of whom gave informed consent to the experimental procedure which was approved by the local Ethical Committee. In two cases the experiments were repeated 3 and 4 times. The subjects were comfortably seated in an armchair. Electromyographic activity from the orbicularis oculi muscles was recorded by surface electrodes set

\section{1) Conditioning stimulation}

Single pulses of $0.1-0.5 \mathrm{~ms}$ duration were given through bipolar surface electrodes, the cathode being proximal to three different nerves: the TR, the $M N$ and SR nerves. Since it was not possible to record the incoming volley, stimulus strength was expressed in multiples ( $x$ ) of the perception $(P)$ threshold 
(Th). In all cases conditioning strength was kept below the Th for reflex responses. According to the ascending method of limits, ${ }^{23}$ stimuli were increased in $0.25 \mathrm{~mA}$ steps and the reflex Th was the intensity value at which a response was evoked by approximately $50 \%$ of the stimuli. The ascending procedure was also used to estimate the subjective PTh, the value of which was verified several times during each experimental session. Branch I of the TR nerve was stimulated at the supraorbital foramen (supraorbital nerve) and branch III at the mental foramen (mandibular nerve). The $\mathrm{MN}$ nerve was stimulated at the second and third digits (in one case also at wrist level). The SR nerve was stimulated at the lateral malleolus.

\section{2) Test stimulation}

The $R 1$ and $R 2$ responses of the trigeminofacial reflex were evoked by test stimuli $(0.1-0.5 \mathrm{~ms}$ duration) applied by surface electrodes to the supraorbital nerve.

Since the aim of this study was primarily to give a description of the excitability changes of the trigemino-facial reflex, it was important, as a first step, to verify whether the size of the test reflex itself could affect its susceptibility to the conditioning volley. The amount of facilitation of the $R 1$ and inhibition of the $R 2$ reflex components of different size were then tested during a constant conditioning input. Conditioning stimulation was applied to the $\mathrm{MN}$ nerve (conditioning-test interval $200 \mathrm{~ms}$, intensity $1.1 \times$ PTh) and the size of the test responses was systematically varied by chang-

Figure 1 Example of the $R 1$ facilitation and $R 2$ inhibition induced by the same conditioning stimulus to the median nerve $(1.1 \times P T h ; 200 \mathrm{~ms}$ $(1 \cdot 1 \times P T h ; 200 \mathrm{~ms}$
conditioning-test interval) when different control sizes were used. Note the large facilitation on $R 1$ response when the conditioning stimulus was applied on a reflex test of small amplitude $(A)$; the same conditioning volley failed to evoke such effect when applied on a test reflex the size of which was kept near its maximum value (B).

\section{0uvL L}
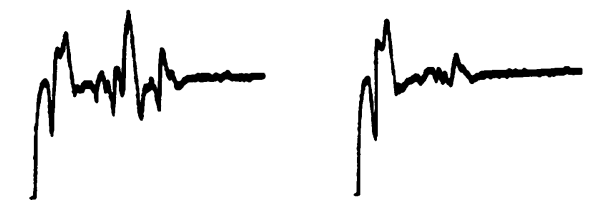

Figure 2 Susceptibility to a constant conditioning stimulus of the R1 (open circles) and $R 2$ (filled circles) test responses of different sizes. Conditioning stimulation as in fig 1. The amount of the effects on the conditioned responses was plotted against the control reflexes size. Each symbol is the mean of ten measurements. Vertical bars $1 S D$ of mean. ing the strength of the test stimulus to the supraorbital nerve. Figure 1 shows a representative example of the effect evoked by the same conditioning stimulus on $R 1$ and $R 2$ responses of small (A) and large size (B). It is apparent that the facilitation of $\mathrm{Rl}$ response is much more evident when the size of the test reflex is small. Figure 2 gives a detailed description of the susceptibility changes of $R \mathbf{l}$ and $R 2$ responses in relation to their control size. The amount of $\mathrm{Rl}$ facilitation and $\mathrm{R} 2$ inhibition (expressed as a percentage of their control values) is plotted against the size of the test reflex (as a percentage of its maximum value) giving a two-part curve: 1) at low reflex amplitude (below $50 \%$ of their maximum value) the susceptibility to the conditioning volley was high for both $R 1$ and R2 reflexes; 2) with increasing unconditioned reflex size, the amount of the $\mathrm{R} 2$ depression remained almost constant, the curves exhibiting a plateau. On the contrary, when the size of the $R l$ response approached its maximum value (above $70-80 \%$ ), no facilitatory event was apparent, suggesting saturation of the response. Accordingly the intensity of the test stimulus was adjusted to evoke an $\mathrm{Rl}$ response $50-70 \%$ of its maximum size. This allowed: a) acceptably stable responses; b) sufficient margin to observe any phenomena of facilitation; c) an R2 response within the plateau phase of the curve. To disclose better the time course of the $\mathbf{R} \mathbf{l}$ facilitation, the size of the test response was occasionally' adjusted to its lower value (20-30\% of its maximum value). This resulted in a large scatter of the data points necessitating several trials before the temporal profile of facilitation could be unambiguously recognized.

\section{3) General experimental procedure}

The stimulus sequences were regularly alternated as follows: 1) control test reflexes alone; 2) test stimulus preceded by conditioning stimulation. Two groups of experiments were performed: 1) keeping the strength of the conditioning stimulus constant (below reflex threshold), the conditioning-test interval was randomly varied from 10 to $400-900 \mathrm{~ms} ; 2$ ) keeping the conditioning-test interval constant, the strength of the conditioning stimulus was randomly varied from $0.7-0.8$ to $1 \cdot 2-1.5$ $\times$ PTh. The sequence, unconditioned-conditioned responses, was repeated 10-15 times for each conditioning-test interval and for each conditioning stimulus strength. Stimuli were given at intervals of $15-20 \mathrm{~s}$, delivered by constant-current stimulator. The EMG signals were fed from the oscilloscope to a full wave rectifier and further to an averager and integrator. The digital integral values of $R 1$ and $R 2$ conditioned reflex components were measured and expressed as a percentage of their unconditioned values.

\section{Results}

To obtain a valid comparison between the effects of different cutaneous conditioning stimuli on TR reflex responses, all the data 
illustrated here are from the same subject. Although the amount of facilitation and/or inhibition caused by the various conditioning stimuli was variable from one subject to another, qualitatively similar results were obtained in all cases.

The time course of the $R 1$ and $R 2$ responses when preceded by subliminal supraorbital nerve stimulation $(1.0 \times \mathrm{PTh})$ is shown in fig 3. The early $R l$ response showed a facilitatory phase between 30 and $60 \mathrm{~ms}$, followed by a long lasting inhibition which was maximal for a conditioning-test interval of $100-150 \mathrm{~ms}$. The

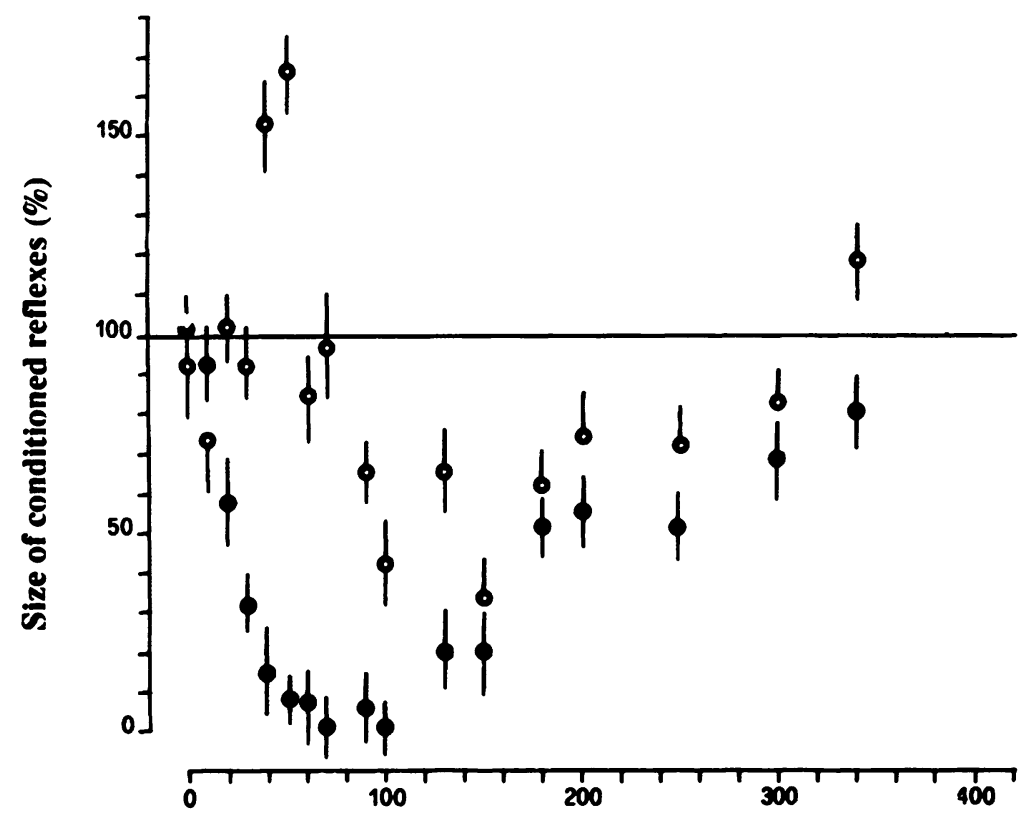

Conditioning-test interval (ms)

Figure 3 Time course of the effects of a conditioning stimulus applied to the ipsilateral supraorbital nerve (1.0 $\times$ PTh) on $R 1$ (open circles) and $R 2$ (filled circles) test reflex responses. The size of the conditioned $R 1$ and $R 2$ responses is expressed as a percentage of their unconditioned values. Each symbol is the mean of ten responses. Vertical bars $1 S D$ of mean.
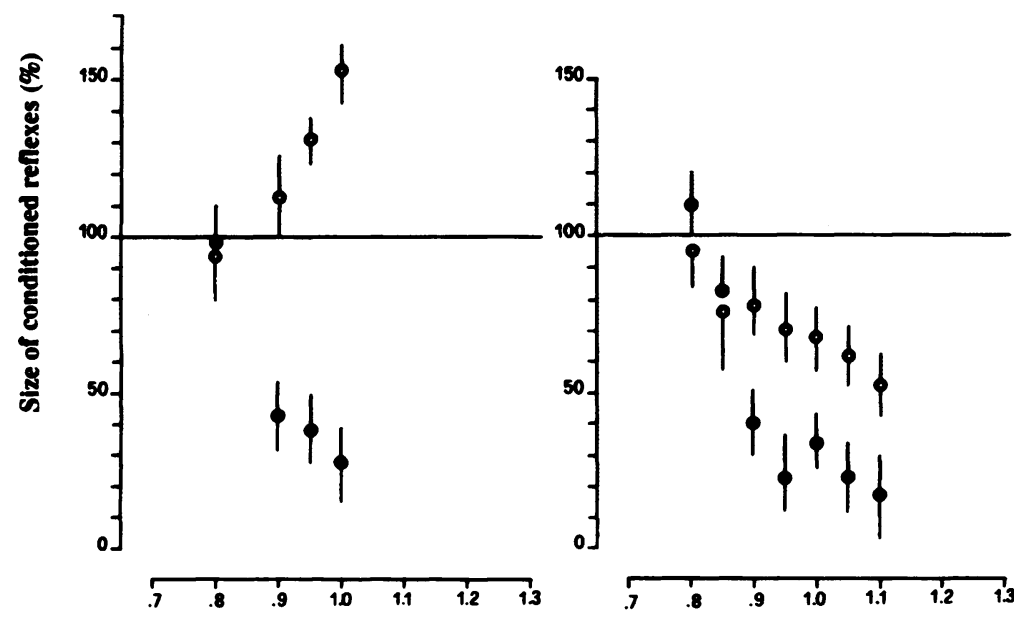

Intensity of conditioning stimulus (xPTh)

Figure 4 Effect of changing the strength of conditioning stimuli applied to the ipsilateral supraorbital nerve on $R 1$ (open circles) and $R 2$ (filled circles) test responses. The conditioning-test interval was kept constant at $50 \mathrm{~ms}$ (on the left) and $100 \mathrm{~ms}$ (on the right). Each symbol is the mean of ten responses. Vertical bars 1 SD of mean. size of the conditioned response recovered to near its control value within $300-400 \mathrm{~ms}$. The same conditioning stimulation resulted in a monotonic long-lasting inhibition of the late R2 response. The minimum effective conditioning-test interval was $15-20 \mathrm{~ms}$. The test response was maximally depressed for an interval of $100-150 \mathrm{~ms}$ and returned to its control level for intervals of $300-400 \mathrm{~ms}$. A comparable time course was obtained for the controlateral R2 response. Very similar effects on the $\mathbf{R} 1$ and $\mathbf{R} 2$ responses from the orbicularis oculi muscle were also obtained when the conditioning stimulus $(1.0 \times \mathrm{PTh})$ was applied to the ipsilateral mental nerve (not illustrated). In an attempt to determine the $T h$ of these changes and whether the same afferent fibres were responsible for both excitatory and inhibitory variations in the test reflexes, a conditioning stimulus of increasing strength was applied at $50 \mathrm{~ms}$ (corresponding to the increasing phase of the $\mathbf{R} \mathbf{l}$ and the decreasing phase of R2) and $100 \mathrm{~ms}$ (corresponding to the inhibition of both responses) of interstimulus delay (fig 4). With a delay of $50 \mathrm{~ms}$, increasing the strength of the TR nerve stimulation resulted in a progressive increase in $R \mathbf{l}$ and decrease in R2, both appearing at $0.9 \times$ PTh. When the interstimulus delay was fixed at $100 \mathrm{~ms}$ the depression of both $\mathrm{Rl}$ and $\mathrm{R} 2$ responses was also apparent at a conditioning strength of $0.9 \times \mathrm{PTh}$ [mean (SD) 0.92 (0.02); range 0.90-0.95 $\times$ PTh]. That the Th values were identical suggests that these changes are contingent upon the same low-threshold afferent fibres. Figure 5 illustrates the results obtained when the conditioning stimulus was applied to the $M N$ nerve fibres arising from the fingers $(1.0 \times \mathrm{PTh})$. At interstimulus interval longer than 25-30 ms, the Rl component showed a first facilitatory peak followed by a subsequent long lasting facilitation which recovered at 500-600 ms. A similar but opposite time course was shown by the $R 2$ reflex response. In the same subject the conditioning

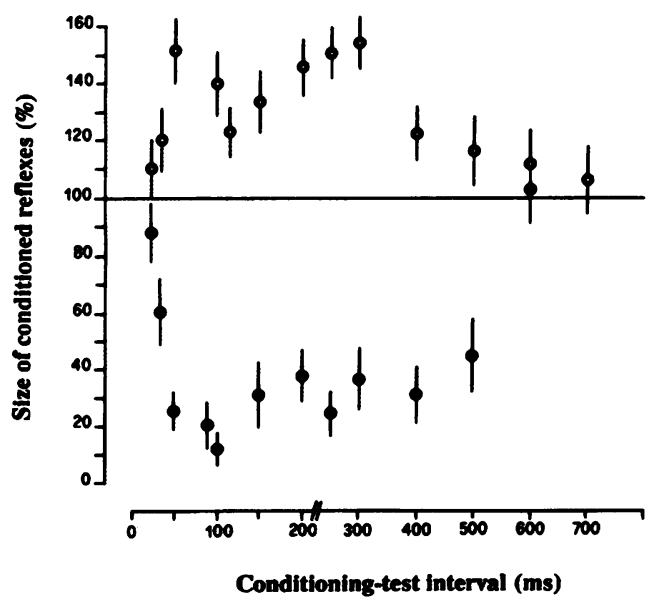

Figure 5 Time course of the effects of a conditioning stimulus applied to the MN nerve $(1.0 \times P T h$ to ipsilateral II-III digit) on R1 (open circles) and R2 (filled circles) test reflex responses. The size of the conditioned $R 1$ and $R 2$ responses is expressed as a percentage of their unconditioned values. Each symbol is the mean of ten responses. Vertical bars $1 S D$ of mean. 


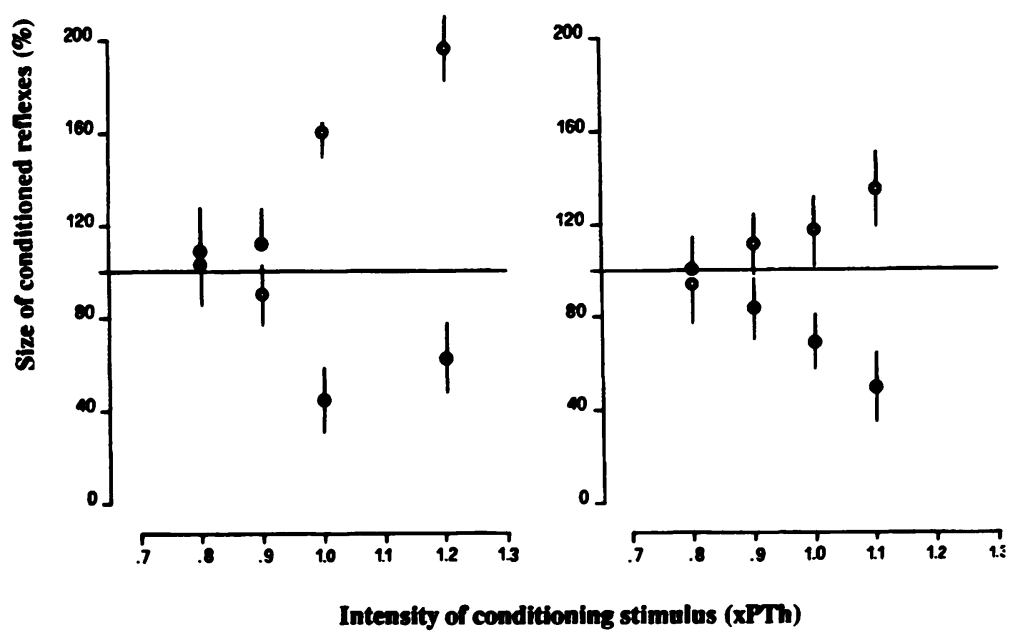

Figure 6 Effect of changing the strength of conditioning stimuli applied to the MN nerve (ipsilateral II-III digit) (on the left) and SR nerve (on the right) on R1 (open circles) and $R 2$ (filled circles) test reflex responses. Conditioning-test interval was kept constant at 200 ms. Each symbol is the mean of ten responses. Vertical bars 1 SD of mean.

stimulation was also applied to the $M N$ nerve at the wrist. The intensity was set at the motor threshold (2.5 $\times$ PTh in this case) to ensure that, in addition to the cutaneous volley, a large fraction of muscular afferents was also activated. Virtually identical results were obtained, although the recovery phase of the $\mathrm{R} 2$ response remained incomplete $(60-70 \%$ of its control size) up to $900-1000$ ms. Figure 6 (left panel) shows the effects of increasing conditioning stimuli applied to the second and third digits, at $200 \mathrm{~ms}$ of interstimulus delay. Both the Rl facilitation and $R 2$ inhibition became apparent at $1.0 \times \mathrm{PTh}$ [mean (SD) $0.96(0.03)$; range 0.9-1.0 $\times$ PTh). A similar time course of both these reflex responses was obtained when the conditioning stimulation was applied to the $S R$ nerve at the ankle (not illustrated). A slight long lasting monotonic facilitation of the $\mathrm{Rl}$ response and a deep long lasting monotonic inhibition of the $R 2$ response started at intervals of $40-45 \mathrm{~ms}$ and recovered within $700-800 \mathrm{~ms}$. None of the cases showed an early facilitatory peak of the $R \mathbf{l}$ component. Increasing conditioning stimulus strength (200 ms interstimulus interval), both the inhibitory effect on $\mathrm{Rl}$ and the facilitatory effect on R2 were apparent at $1 \times$ PTh (right panel fig 6) [mean (SD) 1.04 (0.09); range 0.95-1.20 x PTh).

The mean (SD) Th values for the unconditioned $\mathbf{R} 1$ and $R 2$ test responses were respectively $2 \cdot 27 \times$ PTh $[(0 \cdot 7)$; range $1 \cdot 55-3 \cdot 3]$ and $1.46 \times$ PTh [(0.5); range 1-2.45]; the absolute mean (SD) value of PTh was $1.15 \mathrm{~mA}$ $[(0.5)$, range $0.5-2 \mathrm{~mA}]$.

\section{Discussion}

A single perception threshold conditioning stimulus to the $T R, M N$ and $S R$ nerves modified the excitability of neural pathways mediating the early $R \mathbf{l}$ and late $R 2$ components of the trigemino-facial reflex. Two main factors suggest that these effects depend on the activation of large cutaneous afferent fibres: a) in all cases the threshold of the effects was very low (0.9-1.2 $\times$ PTh), suggesting that only low-threshold afferent fibres were activated; b) when the stimulus was applied to the median nerve, similar effects were observed for stimulation at the wrist (which is supposed to activate both muscular and cutaneous afferents) and for selective stimulation of the cutaneous afferents from the fingers.

Activation of low-threshold TR or limb cutaneous afferent fibres produced a monotonic long-lasting depression of the $\mathrm{R} 2$ reflex component. Following activation of TR nerve, this inhibition was apparent at a conditioningtest interval of $15-20 \mathrm{~ms}$, reached a maximum at $100 \mathrm{~ms}$ and lasted $300-400 \mathrm{~ms}$.

When the conditioning stimulus was applied to the $M N$ and $S R$ nerve afferents, the inhibition had a longer latency (25-30 ms and 40-45 ms respectively) and lasted $500-600 \mathrm{~ms}$. The greater afferent delay due to the distance between the site of application of the stimulus and the brainstem in which interactions between conditioning and test volleys are presumed to occur (see below), can explain the later onset of inhibition. In addition, the time dispersion of the arrival of action potentials in the afferent fibres from $M D$ and SR nerves must be rather large with the long conductance distances that are involved. This time dispersion of the conditioning spikes could be an additional contributory factor responsible for the longer time course of the R2 inhibition with respect to that observed after TR stimulation.

The almost identical temporal trajectory of the R2 inhibition originating from TR, MN and $S R$ nerves suggests a common mechanism. It has been shown in animal studies that impulses in the TR fibres or in ascending and descending pathways in relation to the $T R$ complex, can depress the TR second order neuron response evoked by stimulation of the TR afferent fibres. ${ }^{24-29}$ For the ascending pathways it has been shown in animals that stimulation of the posterior columns or the superficial radial and sciatic nerves is effective in producing depression in the TR nuclei. ${ }^{24} 2730$ Ascending influences from limb afferents on TR reflex responses have also been observed in humans. ${ }^{17}$ Neural elements of the medullary and upper spinal cord reticular formation may be a possible common final pathway of these inhibitory interactions. ${ }^{273132}$ Much evidence exists that presynaptic depolarisation of the TR primary afferents largely contributes to the inhibition observed within TR nuclei. ${ }^{242528-3033-35}$ It is now generally accepted that this depression is caused by an increase in conductance of the primary afferent terminals brought about by axo-axonal TR synapses, ${ }^{29}$ resulting in reduced transmitter release. ${ }^{36-37}$ In addition to any post-synaptic inhibitory mechanism which might be operating, we believe that presynaptic inhibition was also the primary factor contributing to the inhibition of the R2 reflex component. Two main arguments favour this possibility: a) the time course of this inhibition is identical to the time course of presynaptic inhibition described 
in animals and human spinal cord; ${ }^{38}$ b) there is a surprising identity between the time course of the R2 inhibition observed here, and that of the TR primary afferent depolarisation seen in the cat. 242729

In contrast with the monotonic inhibition of $R 2$, the early $R 1$ reflex component undergoes more complex variations. When the test stimulus applied to the supraorbital nerve was preceded by a conditioning stimulus to the TR fibres, (branch I or III) a facilitatory phase, having a maximum between 40 and $50 \mathrm{~ms}$, was observed, cut off by a subsequent long-lasting inhibition which paralleled the time course of the R2 depression (fig 2). In the cat, a facilitatory phase, with a maximum at about $30 \mathrm{~ms}$, has been observed on facial motor neurons following stimulation of trigeminal afferents. ${ }^{39} \mathrm{~A}$ similar motor neuronal facilitation may account for the present results. Since the latency of this phase appears to coincide with the time at which the $R 2$ component is expected, this facilitation may be produced by the subliminal activation, brought about by the conditioning volley, of the R2 reflex pathway inducing EPSPs on facial motor neurons. Indeed, since the Th of the $R 2$ response is very close to the PTh (that is below the Th of the Rl reflex), it is conceivable that the same lowthreshold afferent fibres activated by the subliminal conditioning stimulus are also responsible (when adequate spatial and/or temporal summation takes place) for the late R2 response. In the cat this short latency excitatory phase is followed by a period of reduced responsiveness of motor neurons lasting 30-100 ms. ${ }^{39}$ Because of the experimental condition used, that inhibition may be partially due to motor neurone after-hyperpolarisation following the conditioning reflex response: when the motor neurone is fired in the conditioning volley the subsequent afterhyperpolarisation depresses its excitability and prevents it from firing again. This, however, seems unlikely to account for our case, since the conditioning stimulus was constantly kept below the motor Th, thus ruling out any postspike after-hyperpolarisation. As shown in fig 3 , the temporal profile of the $\mathbf{R l}$ depression parallels that of the $R 2$ component, except for a later onset, probably masked by the preceding facilitation. By a process of analogy, therefore, the most obvious possibility is that it is presynaptic in origin.

When the conditioning stimulus was applied to the $M N$ and $S R$ afferents, the $R \mathbf{l}$ component showed a slight long-lasting facilitation with a time course similar to that of the R2 inhibition (fig 5). Most or all of the presynaptic inhibitory pathways are capable of exerting not only transient but also tonic inhibitory influences on the primary afferent terminals. Temporary removal of tonic presynaptic depolarisation would lead to transitory disinhibition, which could be recorded as facilitation. For example, removal of tonic presynaptic inhibition on Ia fibres restores the monosynaptic excitatory post-synaptic potential to its control height. ${ }^{40}$

Electrophysiological evidence also exists in favour of a tonic presynaptic action on TR primary afferents. ${ }^{29354142}$ In view of this and of the evidence that $\mathrm{Rl}$ facilitation has a time course very similar to that of $R 2$ inhibition, one of the possibilities is that this long-lasting facilitation is due to a transient reduction in tonic presynaptic control acting on the TR afferents responsible for $\mathrm{R} l$ reflex. However, a prerequisite for such a theory is that TR primary afferents for the $\mathrm{Rl}$ response are different from those responsible for the R2 component. The difference between $R \mathbf{l}$ and $R 2$ Th values observed here (see Results Section) and in previous studies ${ }^{23}$ suggests that the $R 1$ component is contingent upon TR afferents smaller (less excitable fibres) than those projecting onto the $R 2$ pathway.

Close observation of fig 5 shows that the facilitation of $\mathbf{R}$ appears to have an early peak which roughly coincides with the phase of short latency facilitation observed after TR stimulation (fig 3). Similarly, this phase of facilitation may be due to the subliminal activation of facial motor neurons induced by the conditioning stimulation of the $\mathrm{MN}$ nerve. It has been shown that $\mathrm{MN}$ afferents access facial motor neurons generating, under appropriate condition, a reflex response in the facial muscles. ${ }^{43}$ This interpretation is indirectly sustained by evidence that the same facilitatory peak does not appear for stimulation of the SR nerve, which never evokes facial reflex responses even at high stimulus intensity.

Our results with limb stimulation seem compatible with those of Bulou et al, ${ }^{17}$ who used the radial and SR as conditioning nerves (see fig 3). Their observations become clearer as a result of this study: first, it demonstrates that large cutaneous afferents make a major contribution to these TR reflex changes; second, in view of the similarity of the reflex changes produced, TR and limb afferents appear to operate via a common neural substrate. A similar conclusion was also reached by Rimpel et al $^{44}$ who observed almost identical effects of acoustic and visual stimuli on TR test responses.

Figure 7 shows a schematic model of hypothetical presynaptic control of the pathways mediating the $R 1$ and $R 2$ reflex components. $A$ conditioning volley in low threshold TR afferents (Ltta) subliminally activates the pathway responsible for the $\mathbf{R} 2$ component, causing an increase in the discharge of presynaptic interneurones $X$ and $Z$ acting on the afferent fibres of both the Rl (Htta) and R2 (Ltta) responses. This is translated into a parallel depression of these reflex responses (fig 3). Incidentally, subliminal activation of the $R 2$ pathway could evoke excitatory post-synaptic potentials in the motor neurons, possibly responsible for the first facilitatory phase observed on the $R \mathbf{l}$ component. Low threshold afferent fibres from limb nerves (Ltla) converge on the interneuron $Z$ but not on $X$. The increased activity of the $Z$ interneuron depresses the R2 pathway, consequently reducing the firing onto presynaptic interneuron X acting on the Rl Htta. This causes disinhibition of these afferents and a consequent facilitation of the $R 1$ component (fig 5). 
Figure 7 A hypothetical model illustrating presynaptic control onto the pathways mediating the $R 1$ and $R 2$ components of the trigemino-facial reflex. Htta: High threshold trigeminal afferents; Ltta: Low threshold trigeminal afferents; Ltla: Low threshold limb afferents; $X$ and $Z$ : last interneurons of putative pathways acting presynaptically on $T R$ primary afferents; $R 1$ : reflex pathway mediating the $R 1$ component; $R 2$ : reflex pathway mediating the $R 2$ component.

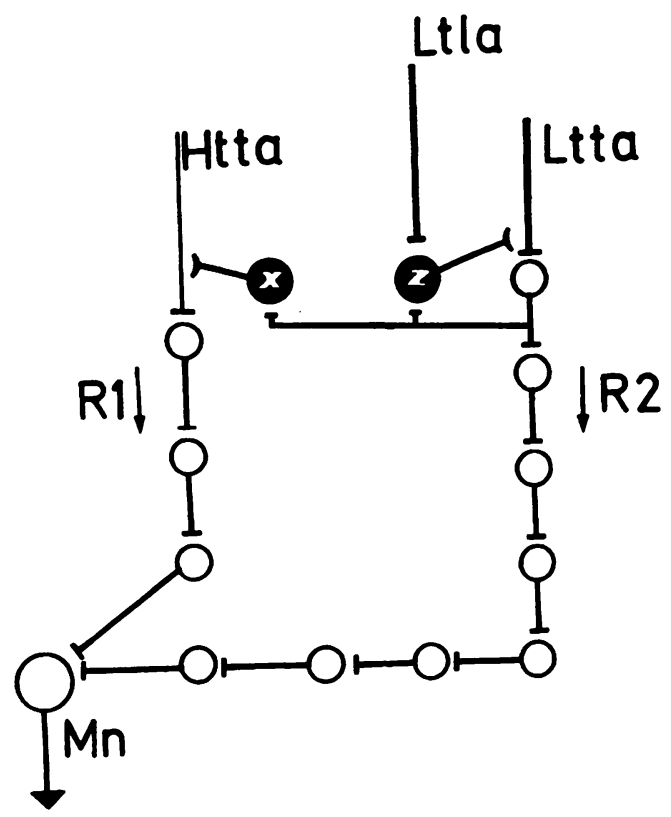

Although our experiments were unable to identify with certainty the neural substrate of the R1 and R2 changes, it is plausible to conclude that presynaptic action from lowthreshold cutaneous afferents on TR terminals is a major contributory factor. The possibility of facilitating or depressing cutaneous transmission at presynaptic level could certainly constitute a powerful means of reducing and selecting peripheral information pertinent to sensory and/or motor processing. For example, "trigeminotrigeminal" and "extratrigeminotrigeminal" inhibitory influences can optimise spatial contrast, respectively within the same receptive field and between different sensory channels, to facilitate the localisation of the stimulus.

A final comment is required on the observation that even a stimulus strength below the subjective PTh is sometimes effective in producing changes in the trigemino-facial reflexes. One possibility is that these low-Th cutaneous afferent fibres do not access the cerebral cortex. However, the evidence in the cat that even the activation of single fibre (from Pacinian corpuscles) is able to generate a corticol evoked response ${ }^{45}$ argues against this possibility. Sensory perception should not be regarded as depending exclusively on the peripheral afferents activated but also on the temporal and spatial gradients of the applied stimulus.

We wish to express our gratitude to Dr B Decchi for reading and commenting upon the manuscript. This work was supported by grants from the Italian CNR and Regione Toscana.

1 Kugelberg E. Facial reflexes. Brain 1952;75:385-96. 2 Rushworth G. Observation on blink reflexes. I Neurol Neurosurg Psychiatry 1962;25:93-108.

3 Shahani BT, Young RR. Human orbicularis oculi reflexes. Neurology 1972;22:149-54.

4 Rossi B, Risaliti R, Rossi A. The R3 component of the blink reflex in man: a reflex response induced by activation of high threshold cutaneous afferents. Electroencephalogr Clin Neurophysiol 1989;73:334-40.

5 Lindquist Chr, Martensson A. Mechanisms involved in the cat's blink reflex. Acta Physiol Scand 1970;80:149-59.

6 Hiraoka M, Shimamura M. Neural mechanisms of the corneal blinking reflex in cats. Brain Res 1977;125:265-75.
7 Ongerboer de Visser BW, Kuypers HGJM. Late blink reflex changes in lateral medullary lesions: an electrophysiological and neuroanatomical study of Wallenberg's syndrome. Brain 1978;101:285-94.

8 Trontelj MA, Trontelj JV. Reflex arc of the first component of the human blink: a single motoneurone study. $f$ Neurol Neurosurg Psychiatry 1978;41:538-47.

9 Ongerboer De Visser BW, Moffie D. Effects of brainstem and thalamic lesions on the corneal reflex. Brain 1979; 102:595-608.

10 Tamai $Y$, Iwamoto $M$, Tsujmoto T. Pathway of the blink reflex in the brainstem of the cat: interneurons between the trigeminal nuclei and the facial nucleus. Brain Res 1986;380:19-25.

11 Evinger C, Manning KA. A model system for motor learning: adaptive gain control of the blink reflex. Exp Brain Res 1988;70:527-38.

12 Evinger C, Sibony PA, Manning KA, Fiero RA. A pharmacological distinction between the long and short latency pathways of the human blink reflex revealed with tobacco. Exp Brain Res 1988;73:477-80.

13 Kimura J. Disorder of interneurons in Parkinsonism. The orbicularis oculi reflex to paired stimuli. Brain 1973;96:87-96.

14 Caraceni T, Avanzini G, Spreafico R, Negri S, Broggi G, Girotti F. Study of the excitability cycle of the blink reflex in Huntington's chorea. Eur Neurol 1976;14:465-72.

15 Kimura J, Harada O. Recovery curves of the blink reflex during wakefulness and sleep. $f$ Neurol 1976; 213:189-98.

16 Ferguson IT, Lenman JAR, Johnston BB. Habituation of the orbicularis oculi reflex in dementia and dyskinetic states. F Neurol Neurosurg Psychiatry 1978;41:824-8.

17 Boulu Ph, Willer JC, Cambier J. Analyse electrophysiologique du réflexe de clignement chez l'homme: intergique du reflexe de clignement chez l'homme: interactions des afferences sensitives segmentaires et Rev Neurol 1981;137:523-33.

18 Berardelli A, Rothwell JC, Day BL, Marsden CD. Pathophysiology of blefarospasm and oromandibular dystonia. Brain 1985;108:593-609.

19 Rossi B, Giannini C, Siciliano G, Sartucci F. The role of the tactile-pressure afferents in the habituation phenomenon of trigemino-facial reflex. Acta Neurol Scand 1985;72:602-5.

20 Tolosa E, Montserrat L, Baves A. Blink reflex studies in focal dystonias: enhanced excitability of brainstem interneurons in cranial dystonia and spasmodic torticollis. Movement disorders 1988;3:61-9.

21 Valls-Sole J, Tolosa ES. Blink reflex excitability cycle in hemifacial spasm. Neurology 1989;39:1061-6.

22 Hatanaka T, Yasuhara A, Kobayashi Y. Electrically and mechanically elicited blink reflexes in infants and children maturation and recovery curves of blink reflex. Electroencephalogr Clin Neurophysiol 1990;76:39-46.

23 Sanes JN, Foss JA, Ison JR. Conditions that affect the thresholds of the components of the eyeblink reflex in humans. I Neurol Neurosurg Psychiatry 1982;45:543-9.

24 Darian-Smith I. Presynaptic component in the afferent inhibition observed within trigeminal brain-stem nuclei of the cat. F Neurophysiol 1965;28:695-709.

25 Darian-Smith I, Yokota T. Cortically evoked depolarization of trigeminal cutaneous afferent fibers in the cat. $\mathcal{F}$ Neurophysiol 1966;29:170-84.

26 Hammer B, Tarnecki R, Vyklicky $L$, Wiesendanger $M$ Corticofugal control of presynaptic inhibition in the Corticofugal control of presynaptic inhibition in the spinal trigem

27 Stewart DH, King RB. Effect of conditioning stimuli upon evoked potentials in the trigeminal complex. I Neurophysiol 1966;29:442-55.

28 Shende MC, King RB. Excitability changes of trigeminal primary afferent preterminals in brainstem nuclear complex of squirrel monkeys (Saimiri sciureus). F Neurophysio 1967;30:949-63.

29 Stewart DH, Scibetta CJ, King RB. Presynaptic inhibition in the trigeminal relay nuclei. $\boldsymbol{f}$ Neurophysiol 1967;20:135-53.

30 Baldissera F, Broggi G, Mancia M. Depolarization of trigeminal afferents induced by stimulation of brain-stem trigeminal afferents induced by stimulation of brain-
and peripheral nerves. Exp Brain Res 1967;4:1-17.

31 Fox JE, Wolstencroft JH. The reduced responsiveness of neurones in nucleus reticularis gigantocellularis following
their excitation by peripheral nerve stimulation. $\mathcal{F}$ Physiol their excitation by peri

32 Inagaki M, Takeshita K, Nakao S, ShiraishiY, Oikawa T. An electrophysiologically defined trigemino-reticulo-facial pathway related to the blink reflex in the cat. Neurosci Let 1989;96:64-9.

33 Wiesendanger $\mathbf{M}$, Hammer B, Tarnecki R. Cortifugal control of presynaptic inhibition in the spinal trigemina nucleous of the cat. The effect of pyramidotomy and barbiturates. Schweitz Arch Neurol Neurochir Psychia 1967;100:255-76.

34 Rowe MJ, Carmody JJ. Afferent inhibition over the response range of secondary trigeminal neurones. Brain Res 1970;18:371-4.

35 Dubner R, Sessle BJ. Presynaptic excitability changes of primary afferent and corticofugal fibers projecting to trigeminal brain stem nuclei. Exp Neurol 1971; 30:223-38.

36 Eccles JC, Schmidt RF, Willis WD. The mode of operation of the synaptic mechanism producing presynaptic inhibition. 7 Neurophysiol 1963;26:523-38.

37 Rudomin P. Presynaptic inhibition of muscle spindle and 
tendon organ afferents in the mammalian spinal cord. Trends Neurosci 1990;13:499-505.

38 Hultborn H, Meunier S, Morin C, Pierrot-Deseilligny E. Assessing changes in presynaptic inhibition of Ia fibres: a Assessing changes in presynaptic inhibition of la fibres:
study in man and the cat. $₹$ Physiol 1987;389:729-56.

39 Lindquist $\mathrm{Chr}$. Analysis of facial reflex facilitation and inhibition by microelectrode recording from the brain inhibition by microelectrode recording f
stem. Acta Physiol Scand 1972;85:183-92.

40 Lund S, Lundberg A, Vyklicky L. Inhibitory action from the flexor reflex afferents on trasmission to Ia afferents. Acta Physiol Scand 1965;6:345-55.

41 Scibetta CJ, King RB. Hyperpolarizing influence of trigeminal nucleus caudalis on primary afferent preterminals in trigeminal nucleus oralis. $f$ Neurophysiol 1969 ; 32:229-38.
42 Schmidt RF. Control of the access of afferent activity to somatosensory pathways. In: Iggo A. ed, vol II. Somatosensory system. Handbook of sensory physiology. Berlin: sensory system. Handbook

43 Dehen H, Bathien N, Cambier J. Le- réflexe palmomentonnier: étude électrophysiologique. Rev Neurol mentonnier: étude

44 Rimpel J, Geyer D, Hopf HC. Changes in the blink responses to combined trigeminal, acoustic and visual repetitive stimulation, studied in the human subject. Electroencephalogr Clin Neurophysiol 1982;54:552-60.

45 McIntyre AK, Holman ME, Veale JL. Cortical responses to impulses from single Pacinian corpuscles in the cat's hind limb. Exp Brain Res 1967;4:243-55.

\section{Neurological stamp}

\section{Niels Stensen (or Steno) 1648-86}

Niels Stensen was still a student when he discovered in 1661 the excretory duct of the parotid gland in sheep. This was later identified in humans by Sylvius. Stensen was the first to identify the heart as a muscle and to recognise the congenital cardiac defects later known as the tetralogy of Fallot. Stensen identified the cerebral grey and white matter and argued that it was idle to speculate about cerebral function when so little was known about its structure. He disagreed with the views of Willis on the location of certain higher functions such as memory, and of Descartes who considered the pineal gland to be the location of the soul that existed only in humans. Stensen showed that the pineal gland existed in other animals. He opposed the views of Borelli who believed that increased muscle bulk noted on contraction was due to a fermentation process generated by a discharge of liquid from the nerves.

Stensen was one of the founders of geology and he wrote important works on the production of strata, fossils and other geological formations. Brought up a Lutheran, Stensen converted to Catholicism in 1667 and gave up the study of science after he was ordained a bishop in 1677 . He was one of the greatest intellects of his time, but died in extreme poverty.

Denmark honoured him with this stamp in 1969 on the 300th anniversary of the publication of his geological work On Solid Bodies (Stanley Gibbons 507, Scott 462).

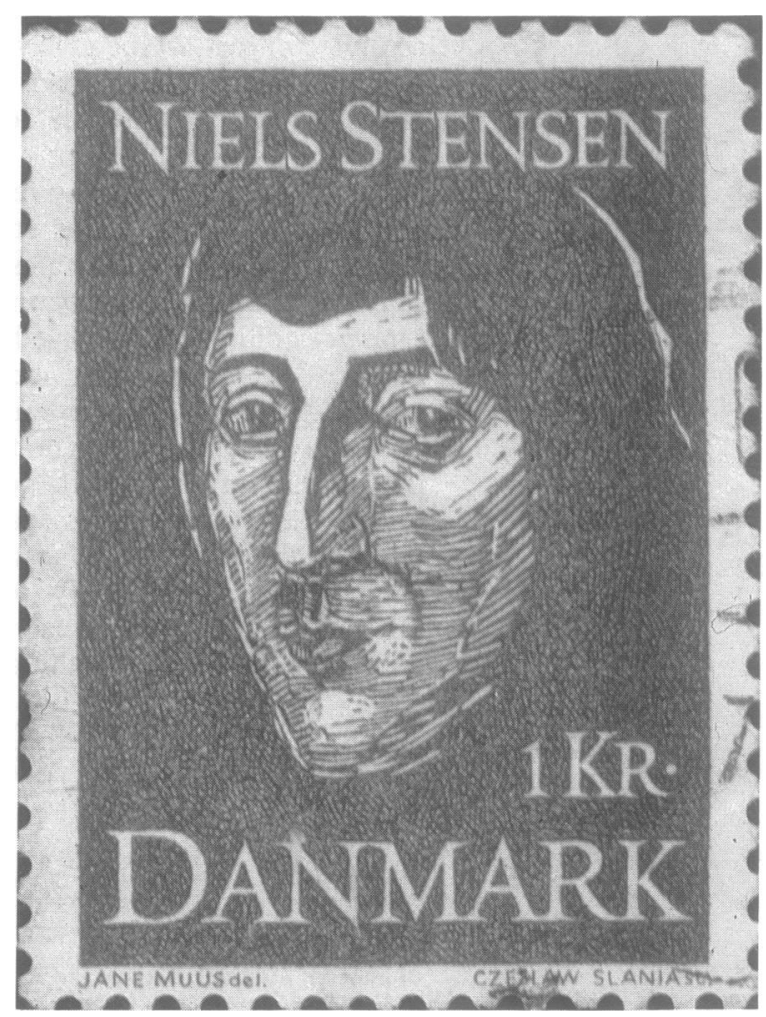

